\title{
Instantaneous Spreading and Einstein Causality in Quantum Theory 1
}

\author{
Gerhard C. Hegerfeldt \\ Institut für Theoretische Physik \\ Universität Göttingen \\ Bunsenstr. 9 \\ 37073 Göttingen, Germany
}

\begin{abstract}
In nonrelativistic quantum mechanics the wave-function of a free particle which initially is in a finite volume immediately spreads to infinity. In a nonrelativistic theory this is of no concern, but we show that the same instantaneous spreading can occur in relativistic quantum theory and that transition probabilities in widely separated systems may instantaneously become nonzero. We discuss how this affects Einstein causality.
\end{abstract}

\section{Introduction}

The principle of the limiting role of the velocity of light is often called 'Einstein causality' [1]. Einstein's principle of finite signal velocity is of fundamental importance for the foundations of physics, both in classical as well as in quantum physics.

Now consider nonrelativistic quantum mechanics and a free particle whose wave function at time $t=0$ vanishes outside some finite region $V$. The latter implies that the particle is in $V$ with probability 1 , and thus with certainty. The free nonrelativistic time evolution is then such that at an arbitrarily short time later the wave function is nonzero arbitrarily far away from the original region $V$ [2]. Thus the wave function instantaneously spreads to infinity and the probability of finding the particle arbitrarily far away from the initial region is nonzero for any $t>0$. But since this superluminal propagation happens in a nonrelativistic theory it is of no great concern.

Relativistic wave equations which are used to describe free relativistic particles are hyperbolic and therefore the propagation is at most luminal so that the above problem does not seem to arise. However, if a wave function $\varphi(\mathbf{x}, t)$ satisfies a relativistic wave equation then the connection between the variable $\mathbf{x}$ and the position of the particle is in general not clear-cut. In fact, the position operator

\footnotetext{
${ }^{1}$ Talk given at the workshop Superluminal (?) Velocities, Cologne, June 6-10, 1998. A talk with a similar content was also given by the author at the symposium Uncertain Reality, New Delhi, January 5-9, 1998.
} 
proposed by Newton and Wigner [3] is not multiplication by $\mathbf{x}$ but rather is a nonlocal function of $\mathbf{x}$. It was noted by Weidlich and Mitra [4] and by Fleming [5] that with the Newton-Wigner position operator superluminal propagation can occur in the localization of a free relativistic particle. Amrein [6] pointed out that this happened also for a proposed position operator for the photon. A similar phenomenon occurred in some models in which localization was expressed by means of a current-density four-vector as shown by Gerlach, Gromes, and Petzold [7].

In 1974 the present author [8] showed that this phenomenon of instantaneous spreading is quite general for a free relativistic particle, irrespective of the particular notion of localization, be it in the sense of Newton-Wigner or others. Later an alternative proof of this result was given by Skagerstam [9], and it was extended to relativistic systems by Perez and Wilde [10 and to quite general, not necessarily relativistic, interactions by Ruijsenaars and the author [11]. The upshot of Ref. [11] was that this instantaneous spreading is mainly due to positivity of the energy plus translation invariance. Neither relativity nor field theory is needed. It was recently shown by the author [12] that translation invariance is also not needed. Hilbert space and positivity of the Hamiltonian (energy) suffices to ensure either instantaneous spreading or, alternatively, confinement in a fixed region for all times.

A further extension was given by the author [13] for free relativistic particles and for relativistic systems which have exponentially bounded tails in their localization outside some region $V$. It was shown that the state spreads out to infinity faster than allowed by a probability flow with finite propagation speed.

In the following we give a brief proof of our basic result of Ref. [8]; for purely pedagogical reasons we use relativistic invariance there. We then present an analysis of Fermi's two-atom system [14] where we follow our Refs. [15, 16]. A large portion will be devoted to a discussion what these results mean for Einstein causality. Probably the most astonishing fact about our results is that so little is needed to derive them. No field theory, no relativity, only Hilbert space and positivity of the energy is needed.

\section{$2 \quad$ Spreading for particles}

If $\psi_{t}(\mathbf{x})$ denotes a wave function of a particle in nonrelativistic quantum mechanics then the probability, $P_{\psi_{t}}(V)$, of finding the particle in a region $V$ at time $t$ is given by

$$
P_{\psi_{t}}(V)=\int_{V}\left|\psi_{t}(\mathbf{x})\right|^{2} d^{3} x \equiv\left\langle\psi_{t}, \chi_{V} \psi_{t}\right\rangle
$$

where $\chi_{V}(\mathbf{x})$ is the characteristic function of $V$ and equals 1 in $V$ and 0 outside. Regarded as a multiplication operator, $\chi_{V}$ is a projector of the spectral 
decomposition of the usual nonrelativistic position operator $\mathbf{X}$. For the NewtonWigner position operators there are analogous projection operators which determine $P_{\psi_{t}}(V)$, but which are no longer given by a simple multiplication by a function.

In this section no position operator will be assumed nor any form of projection operators. But rather I will assume just the existence of some operator $N(V)$ such that the probability $P_{\psi_{t}}(V)$ of finding the particle in $V$ at time $t$ is given by

$$
P_{\psi_{t}}(V)=\left\langle\psi_{t}, N(V) \psi_{t}\right\rangle
$$

where now $\psi_{t}$ is the (abstract) state vector of the free relativistic particle. Because probabilities lie between 0 and 1 one must have

$$
0 \leq N(V) \leq 1
$$

This implies in particular that $N(V)$ is hermitian and that $N(V)^{1 / 2}$ exists as a hermitian positive operator. No explicit form of $N(V)$ will be assumed in the following.

The time development of the free particle is described by the generator $P^{0}=$ $\sqrt{\mathbf{P}+m^{2}}$ for time translation in an irreducible representation of the Poincaré group for arbitrary mass $m \geq 0$ and spin $s=0, \frac{1}{2}, \ldots$. By

$$
U(\mathbf{a})=e^{-i \mathbf{P} \cdot \mathbf{a} / \hbar}
$$

we denote the operator for spatial translations. By translation covariance, the probability of finding the translated state $U(\mathbf{a}) \psi_{t}$ in the translated region $V_{\mathbf{a}}$ is the same as that of finding $\psi_{t}$ in $V$. Replacing $\psi_{t}$ by $\psi_{t}^{\prime} \equiv U(-\mathbf{a}) \psi_{t}$ the last statement implies

$$
P_{\psi_{t}}\left(V_{\mathbf{a}}\right)=\left\langle\psi_{t}, U(\mathbf{a}) N(V) U(\mathbf{a})^{\dagger} \psi_{t}\right\rangle
$$

Note that the r.h.s. of this can be written as a vector norm. We can now prove the following results on instantaneous spreading.

Theorem 1 [8]. Consider a free relativistic particle of positive or zero mass and arbitrary spin. Assume that at time $t=0$ the particle is localized with probability 1 in a bounded region $V$. Then there is a nonzero probability of finding the particle arbitrarily far away at any later time.

Proof: The proof is by contradiction. Let $t>0$ be arbitrary but fixed and assume that at time $t$ the particle is localized with probability 1 in some large, but finite, region containing $V$. This implies that for all sufficiently large $|\mathbf{a}|$, larger than some constant $R_{t}$ say, one has $P_{\psi_{t}}\left(V_{\mathbf{a}}\right)=0$, as indicated in Fig. 1. Eq. (5) then becomes 


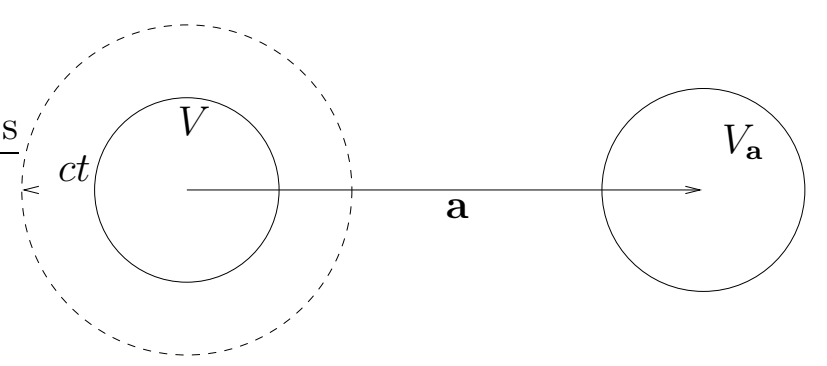

Figure 1: Maximal localization region at time $t$ has no intersection with $V_{\mathbf{a}}$.

$$
\left\|N(V)^{1 / 2} U(\mathbf{a})^{\dagger} \psi_{t}\right\|^{2}=0 \text { for all }|\mathbf{a}|>R_{t} .
$$

Thus the vector inside the norm vanishes. Multiplying the vector by $N(V)^{1 / 2}$ one obtains

$$
N(V) U(\mathbf{a})^{\dagger} \psi_{t}=0 \text { for all }|\mathbf{a}|>R_{t} .
$$

Taking the scalar product of this with $\psi_{0}$ yields

$$
\left\langle N(V) \psi_{0}, U(-\mathbf{a}) \psi_{t}\right\rangle=0 \text { for }|\mathbf{a}|>R_{t} .
$$

We now introduce the abbreviations

$$
\begin{aligned}
\phi & \equiv N(V) \psi_{0} \\
f_{t}(\mathbf{x}) & \equiv\left\langle\phi, U(-\mathbf{x}) \psi_{t}\right\rangle .
\end{aligned}
$$

In momentum space $\mathbf{P}$ becomes multiplication by $\mathbf{p}$ and thus the r.h.s. of Eq. (9) can be written as

$$
f_{t}(\mathbf{x})=\int \underbrace{\frac{d^{3} p}{\left(p^{2}+m^{2}\right)^{1 / 2}} \phi(\mathbf{p})^{\star} \cdot \boldsymbol{\psi}_{0}(\mathbf{p}) e^{-i \sqrt{\mathbf{p}^{2}+m^{2}} t}} e^{i \mathbf{p} \cdot \mathbf{x}}
$$

where a summation over spin variables is understood and where $m=0$ is allowed.

Since, by Eq. (7), $f_{t}(\boldsymbol{x})$ vanishes outside the ball of radius $R_{t}$ around the origin (which means that $f_{t}$ has compact support) its Fourier transform must be analytic, both for $t=0$ and the fixed $t>0$ under consideration. But it is evident from Eq. (10) that the Fourier transform of $f_{t}(\boldsymbol{x})$ is the expression over the brace. However, because of the root in the exponent this expression cannot be analytic in $\mathbf{p}$ for two distinct values of $t$ unless $f_{t} \equiv 0$. For $t=0$ and $\boldsymbol{x}=0$ the latter would imply

$$
\left\langle N(V) \psi_{0}, \psi_{0}\right\rangle=0,
$$

which is a contradiction. This concludes the proof. 
It is worthwhile to point out that Theorem 1 carries over to unbounded regions $V$ like infinite slabs of the form $\left\{(\boldsymbol{x}) ;\left|x_{1}\right| \leq d\right\}$ and that the spreading is indeed over all space ('no holes') and relativity is not needed, as shown by the author and Ruijsenaars [11. Recently the author 12] showed the stronger result that Hilbert space and positivity of the energy (Hamiltonian) yield instantaneous spreading for systems.

In Ref. [13] the author proved a stronger result for exponential tails. We say that a particle is localized with exponentially bounded tails if the probability of finding it outside a ball $B_{r}$ of radius $r$ around the origin, i.e. in $\mathbb{R}^{3} \backslash B_{r}$, decreases at least as $\exp \{-K r\}$ for large $r$, with some $K>m \geq 0$. It was shown that if a particle is thus localized at time $t=0$ then at any later time $t$ one has, for any $c>0$ and any $r_{0}>0$,

$$
P_{\psi_{t}}\left(\mathbb{R}^{3} \backslash B_{r_{0}+c t}\right)>P_{\psi_{0}}\left(\mathbb{R}^{3} \backslash B_{r_{0}}\right)
$$

The strict inequality sign is the main point here. For relativistic systems the same result holds if the tails are bounded Gaussian-like with $\exp \left\{-K^{\prime} r^{2}\right\}$, some $K^{\prime}>0$.

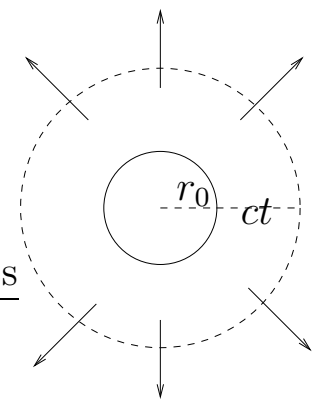

Figure 2: Probability flow of speed at most $c$.

As seen from Fig. 2, if the change of localization probability with time were due to a probability flow of speed at most $c$, then the probability of finding the particle at time $t$ outside the ball $B_{r_{0}+c t}$ could only have grown by the amount between the two spheres and could therefore not exceed that at time $t=0$ outside the ball $B_{r_{0}}$. Just this is violated in Eq. (11).

Eq. (11) yields Theorem 1 if one chooses $V \subset B_{r_{0}}$ and assumes $P_{\psi_{0}}\left(\mathbb{R}^{3} \backslash V\right)=$ 0. Then the r.h.s. of Eq. (11) is zero while the l.h.s. is nonzero so that the probability of finding the particle outside any large ball in nonzero.

\section{The two-atom model of Fermi}

To check finite propagation speed Fermi [14] supposed in his model that by some means one had prepared, at time $t=0$, an atom $A$ in an excited state $\left|e_{A}\right\rangle$ and 
some distance $R$ away an atom $B$ in its ground state, with no photons initially present. The state of the system then develops in time and Fermi calculated the probability to find the state $\left|g_{A}\right\rangle\left|g_{B}\right\rangle\left|0_{\mathrm{ph}}\right\rangle$ at time $t$. He probably had in mind that this only could occur by de-excitation of $A$ accompanied by an emission of a photon and then absorption of the latter by $B$ together with an excitation of $B$. However, actually to check that there are no photons requires, at least in principle, photon measurements over all space. Hence this 'exchange' probability cannot be used for signals, it just refers to statistical correlations. In the spirit of Fermi a better approach would be to calculate the probability of finding $B$ excited, irrespective of the state of $A$ and possible photons. This approach was analyzed by the present author in Refs. [15, 16].

If the observable ' $B$ is in an excited state' makes sense and is represented by an operator $\mathcal{O}_{e_{B}}$ then

$$
\left\langle\psi_{t}, \mathcal{O}_{e_{B}} \psi_{t}\right\rangle
$$

is the probability to find $B$ excited. Again, since probabilities lie between zero and 1 , one must have

$$
0 \leq \mathcal{O}_{e_{B}} \leq 1
$$

For example, $\mathcal{O}_{e_{B}}$ might be a projection operator, as for bare states, but more general possibilities are conceivable. The explicit form of $\mathcal{O}_{e_{b}}$ is not needed, only Eqs. (12) and (13) are used.

To more clearly separate what is physics and what mathematics the next theorem is phrased in purely mathematical terms. Application to physics will be explained subsequently.

Theorem 2 [15. Let the operator $H$ be self-adjoint and bounded from below. Let $\mathcal{O}$ be any operator satisfying

$$
0 \leq \mathcal{O} \leq \text { const }
$$

Let $\psi_{0}$ be any vector and define

$$
\psi_{t} \equiv e^{-i H t} \psi_{0}
$$

Then one of the following two alternatives holds.

(i) $\left\langle\psi_{t}, \mathcal{O} \psi_{t}\right\rangle \neq 0 \quad$ for almost all $t$

(and the set of such $t$ 's is dense and open)

(ii) $\left\langle\psi_{t}, O \psi_{t}\right\rangle \equiv 0 \quad$ for all $t$. 
The proof can be found in Refs. [15, 16]. In the application to Fermi's model $H$ would be the Hamiltonian, $\mathcal{O}=\mathcal{O}_{e_{B}}$ and $\psi_{0}$ would represent the initial physical state in which $A$ is excited, $B$ not, and with no photons so that $\left\langle\psi_{0}, \mathcal{O}_{e_{B}} \psi_{0}\right\rangle=0$. One would then conclude that $B$ is either immediately excited with nonzero probability, or never.

The above mathematical result has been used recently by the author [12 to show that translation invariance is not needed for instantaneous spreading of particles or system. The Hamiltonian can be quite general, only boundedness from below is required, and this ensures either instantaneous spreading or confinement in a fixed bounded region for all times, corresponding to the alternatives (i) and (ii) above.

\section{Spreading in relativistic quantum mechanics}

At first sight the Dirac equation might seem to be a counterexample to our results on instantaneous spreading. Indeed, this wave equation is hyperbolic, implying finite propagation speed. For the localization operator $N(V)$ one might take the characteristic function $\chi_{V}(\boldsymbol{x})$, just as in the nonrelativistic case of Eq. (II) and in contrast to the (nonlocal) Newton-Wigner operator. Then, for a wave function initially vanishing outside a finite region, i.e. of finite support, the localization does evolve with finite propagation speed! Doesn't this contradict Theorem 1 above?

This example is instructive since it shows the importance of the positiveenergy condition. The Dirac equation contains positive and negative energy states, and therefore we conclude from our results that positive-energy solutions of the Dirac equation always have infinite support to begin with! This is phrased as a mathematical result for instance in the book of Thaller [17].

If there are no strictly localized states in the theory then Theorem 1 does not apply! Strict localization of a state $\psi$ in a region $V$ means that $\langle\psi, N(V) \psi\rangle=1$, and this gives

$$
0=\langle\psi,(\mathbf{1}-N(V)) \psi\rangle=\left\|(\mathbf{1}-N(V))^{1 / 2} \psi\right\|^{2}
$$

where the root exists by Eq. (3). Similar to Eqs. (6) and (7) this implies

$$
N(V) \psi=\psi .
$$

Hence $\psi$ is an eigenvector of $N(V)$ for the eigenvalue 1 if $\psi$ is strictly localized in $V$, and vice versa. The eigenvalue 0 means strict localization outside $V$.

The existence or nonexistence of strictly localized states depends on the form of $N(V)$. For example, if one has a self-adjoint position operator $\hat{\boldsymbol{X}}$ with commuting components, then $N(V)$ is a projection operator from the spectral decomposition of $\hat{\boldsymbol{X}}$ and thus has eigenvalues 1 and 0 . Hence in this case there are 
strictly localized states for any region $V$, and Theorem 1 implies instantaneous spreading.

This instantaneous spreading also occurs for position operators with selfadjoint but non-commuting components $\hat{X}_{i}$. Each $\hat{X}_{i}$ has a spectral decomposition whose projection operators give the localization operators for infinite slabs. Eigenvectors for the eigenvalue 1 represent states strictly localized in these slabs, and by the remark after Theorem 1 there is instantaneous spreading in this case, too.

To avoid instantaneous spreading one has therefore to consider localization operators $N(V)$ which are not projectors, for example positive operator-valued measures. However, if one insists on arbitrary good localization, i.e. on tails which drop off arbitrarily fast, then one runs into Eq. (11). This equation can be interpreted as stemming from an infinitely fast probability flow.

If in the Fermi model atoms $A$ and $B$ would develop instantaneous tails then an immediate excitation would not seem surprising. So the two phenomena appear to be connected. Mathematically this connection is of course given by the assumed positivity of the energy and has been discussed in detail by the author in Ref. [12].

Could instantaneous spreading be used for the transmission of signals if it occurred in the framework of relativistic one-particle quantum mechanics? Let us suppose that at time $t=0$ one could prepare an ensemble of strictly localized (non-interacting) particles by laboratory means, e.g. photons in an oven. Then one could open a window and would observe some of them at time $t=\varepsilon$ later on the moon. Or to better proceed by repetition, suppose one could successively prepare strictly localized individual particles in the laboratory. Preferably this should be done with different, distinguishable, particles in order to be sure when a detected particle was originally released. Such a signaling procedure would have very low efficiency but still could be used for synchronization of clocks or, for instance, for betting purposes.

\section{$5 \quad$ Field-theoretic aspects and discussion}

Localization of particles. In field theory difficulties with particle localization have been known for a long time [18]. However, our results are more general since no fields are involved and none of the particular assumptions or axioms of field theory, except for positivity of the energy, are used.

In a field-theoretic context permanent infinite tails can be understood intuitively through clouds of virtual particles due to renormalization ('dressed states'). It is also conceivable that whenever one tries to prepare a localized particle or system one automatically creates particle-antiparticle pairs outside the desired localization region, and this would have the same effect as tails.

Instead of speaking about infinite tails one may also envisage that all particle 
detectors exhibit, for example, inherent noise and that therefore localization with probability 1 or zero can never be recorded. This would essentially lead to the same conclusions as permanent infinite tails.

Fermi's model. From the foregoing discussion it is evident that, in a fieldtheoretic context, excitation of atom $B$ need not be due to absorption of a photon emitted by atom $A$. The excitation could rather be due to vacuum fluctuations, photon clouds etc. Or the excitation maybe just spontaneous, whatever that means. At a more fundamental level, the notion of bounds states has its intricacies in field theory, and the corresponding observables in the Fermi model might be hard to put on a mathematically rigorous footing.

If infinite tails always exist, or if all counters are influenced by vacuum fluctuation, then how can finite propagation speed or Einstein causality be checked at all? Here it is useful for differentiate between two notions of causality.

Strong causality. By this we mean that for each individual experiment in which two systems, separated by a distance $R$, are prepared at time $t=0$ no disturbance or excitation of the second system occurs for $t<R / c$ [16]. This notion is analogous to energy momentum and baryon conservation in each individual scattering process in particle physics. Strong causality would hold in the Fermi model if the transition probability were strictly zero for $t<R / c$. It seems that Fermi had this causality notion in mind. Our results show that strong causality cannot be checked (unless a possible way out via cut-off theories holds [16]), or it may fail in a theory.

Weak causality. This notion was introduced by Schlieder [20], and it means Einstein causality for expectation values or ensemble averages only, not for each individual process. Thus for the above two systems, expectation values for the second system need not vanish for $t<R / c$, but it would take at least a time $t=R / c$ to produce an effect on them. To exhibit this effect it is convenient to subtract possible fluctuations of the second system alone. Theorem 2 does not apply to this situation since this difference is not the expectation value of a positive operator.

The weak assumptions of the above theorems (just Hilbert space and positive energy) will not be enough to prove weak causality. In Refs. 221, 22] the Fermi model has been studied using the methods and approximations of quantum optics. Vacuum fluctuations and virtual photons contribute to the excitation of atom $B$, and once the expectation value of this contribution has been subtracted, the remainder behaves causally, at least within the approximations employed. This just corresponds to the notion of weak causality. In how far this can be measured will be discussed below.

In the framework of quantum field theory it is sometimes simply argued that local commutation or anti-commutation relations must clearly ensure causal behavior, for instance of localized particles. This implicitly presupposes, however, that the relevant operators, e.g. $N(V)$ or $\mathcal{O}_{e_{B}}$-if they exist - are local functions of the fields. It should be recalled that fields do not enter in our formulation, 
and it should be noted that if $N(V)$ is a local function of the fields then the Reeh-Schlieder theorem [19 implies that its vacuum expectation is nonzero.

Within local quantum field theory a rigorous proof of weak causality for local observables has been given by Schlieder [20] and by Buchholz and Yngvason [23], as well as by Neumann and Werner [24] in an alternative algebraic framework. In Ref. 23] it was moreover shown that restrictions of states to local algebras cannot be tested by means of transition probabilities.

How can one check weak causality experimentally? In the Fermi model one would not use a single pair of atoms but rather an ensemble, either by repetition or by simultaneous realization. If in the Fermi case one has $N$ pairs of atoms $A$ and $B$ one would measure at time $t$ how many $B$ atoms are excited. For $N \rightarrow \infty$ their fraction would be given by $\left\langle\psi_{t}, \mathcal{O}_{e_{B}} \psi_{t}\right\rangle$, while for finite $N$ this would hold only approximately, due to statistical fluctuations. Then one would subtract the - either calculated or measured - excitation probability of $B$ without $A$ present. Weak causality asserts that the difference should be zero for $t<R / c$ - but only for $N \rightarrow \infty$, while for finite $N$ there are always fluctuations. Hence in a strict sense, weak Einstein causality can only be checked experimentally for infinite ensembles. This suggests a macroscopic context.

The main point of our results seems to be that instantaneous spreading holds already under amazingly few assumptions. Neither the existence of fields nor the usual axioms of field theory are assumed - the only input is Hilbert space and positivity of the energy. Our results seem to indicate the need for a mechanism like vacuum fluctuations, clouds of virtual particles, particle-antiparticle pairs, spontaneous excitations, or something like that in order to retain Einstein causality. Our results are compatible with quantum field theory which uses much stronger assumptions and in which vacuum fluctuations etc. are present.

\section{References}

[1] Faster-than-light particles ('tachyons') - when used in a direct and straightforward way - could in principle be employed to reverse the order of cause and effect and to influence the past from the present. Cf., e.g., W. Rindler, Introduction to Special Relativity, 2nd Edition, Clarendon, Oxford 1991, p. $17 \mathrm{f}$. This undesirable consequence can be avoided by a sophisticated reinterpretation which makes the emission and absorption of such particles observer dependent. Cf. O. M. Bilaniuk, V. K. Deshpande, and E. C. G. Sudarshan, Am. J. Phys. 30, 718 (1962), and talks at this workshop.

[2] This is easy to show by means of the free propagator, which is given e.g. in M. Reed and B. Simon, Methods of Modern Mathematical Physics, Vol. II, Academic Press, New York 1975. 
[3] T.D. Newton and R.P. Wigner, Rev. Mod. Phys. 21, 400 (1949). Cf. also S.S. Schweber, An Introduction to Relativistic Quantum Field Theory, New York 1961.

[4] W. Weidlich and K. Mitra, Nuovo Cim. 30, 385 (1963)

[5] G.N. Fleming, Phys. Rev. 139, B963 (1965). This was also investigated by S. Schlieder, in Quanten und Felder, edited by H.P. Dürr; Vieweg, Braunschweig 1971.

[6] W. O. Amrein, Helv. phys. Acta 42, 149 (1969)

[7] B. Gerlach, D. Gromes, and J. Petzold, Z.Phys. 202, 401 (1967), 221, 141 (1969); D. Gromes, Z. Phys. 236 (1970)

[8] G.C. Hegerfeldt, Phys. Rev. D 10, 3320 (1974)

[9] B. Skagerstam, Int. J. Theor. Phys. 15, 213 (1976)

[10] J. F. Perez and I.F. Wilde, Phys. Rev. D 16, 315 (1977)

[11] G.C. Hegerfeldt and S.N.M. Ruijsenaars, Phys. Rev. D 22, 337 (1980)

[12] G.C. Hegerfeldt, in: Irreversibility and Causality in Quantum Theory Semigroups and Rigged Hilbert Spaces, edited by A. Bohm, H.-D. Doebner and P. Kielanowski, Lecture Notes in Physics 504, p. 238, Springer (Berlin 1998)

[13] G.C. Hegerfeldt, Phys. Rev. Lett. 54, 2395 (1985)

[14] E. Fermi, Rev. Mod. Phys. 4, 87 (1932); M.I. Shirokov, Yad. Fiz. 4, 1077 (1966) [Sov. J. Nucl. Phys. 4, 774 (1967)]; W. Heitler and S.T. Ma, Proc. R. Ir. Acs. 52, 123 (1949); J. Hamilton, Proc. Phys. Soc. A 62, 12 (1949); M. Fierz, Helv. Phys. Acta 23, 731 (1950); B. Ferretti, In: Old and new Problems in Elementary Particles, edited by G. Puppi (Academic Press, New York, 1968), p. 108; P.W. Milonni and P.L Knight, Phys. Rev. A 10, 1096 (1974); M.I. Shirokov, Sov. Phys. Usp. 21, 345 (1978); M.H. Rubin, Phys. Rev. D 35, 3836 (1987); A.K. Biswas, G. Compagno, G.M. Palma, R. Passante, and R. Persico, Phys. Rev. A 42, 4291 (1990); A. Valentini, Phys. Lett. A 153, 321 (1991)

[15] G.C. Hegerfeldt, Phys. Rev. Lett. 72, 596 (1994)

[16] G.C. Hegerfeldt, in: Nonlinear, deformed and irreversible quantum systems, edited by H.-D. Doebner, V.K. Dobrev, and P. Nattermann, World Scientific 1995 
[17] B. Thaller, The Dirac Equation, Springer, Berlin 1992

[18] A.S. Wightman and S.S. Schweber, Phys. Rev. 98, 812 (1955)

[19] H. Reeh and S. Schlieder, Nuovo Cim. 22, 1051 (1961). This theorem also exploits analyticity but uses, in addition to positivity of the energy, stronger assumptions of field theory, in particular locality.

[20] S. Schlieder, in: Quanten und Felder, H.P. Dürr Ed. (Vieweg, Braunschweig, 1971), p. 145

[21] A. Labarbara and R. Passante, Phys. Lett. 206, 1 (1994)

[22] P.W. Milonni, D.F.V. James, and H. Fearn, Phys. Rev. A 52, 1525 (1995)

[23] D. Buchholz and J. Yngvason, Phys. Rev. Lett. 73, 613 (1994)

[24] H. Neumann and R. Werner, Intern. J. Theor. Phys. 22, 781 (1983) 